\title{
Incorporación de criterios de fragilidad ambiental y riesgo en la planificación territorial de la costa de Chile central ${ }^{1}$
}

\author{
Belisario Andrade², Federico Arenas ${ }^{2}$ y Marcelo Lagos ${ }^{2}$
}

\begin{abstract}
RESUMEN
Se analiza el estado de los instrumentos de planificación territorial vigentes en la zona costera de la Región del Maule (Chile central) y se comparan con unidades espaciales a escala detallada de fragilidad ambiental y riesgo de tsunami. Se constata la precariedad de dichos instrumentos y la escasa incorporación de consideraciones ambientales en los mismos. En este marco se propone una zonificación que permite incorporar las variables ambientales estudiadas en los instrumentos de planificación del espacio costero.
\end{abstract}

Palabras clave: Zona costera, ordenamiento territorial, geomorfología, riesgo, tsunami.

\begin{abstract}
Analysis of the state of territorial planning tools of the coastal zone of the Maule Region (Central Chile) are made and compared with spatial units of a detailed scale of environmental fragility and tsunami risk. Notice is made of the precariousness and scarce incorporation of environmental considerations of these tools. In this sense, a zonification that allows for the incorporation of environmental variables is proposed for the planification tools of the coastal zone.
\end{abstract}

Key words: Coastal zone, territorial planning, geomorphology, risk, tsunami.

Esta comunicación expone consideraciones sobre la necesidad de insertar variables ambientales, en particular bajo una óptica geomorfológica, en la planificación territorial de la Zona Costera (ZC), refiriéndose especialmente a ejemplos de la costa de la Región del Maule (Figura $N^{\circ} 1$ ). La presencia de espacios relativamente poco intervenidos en el área de estudio le otorga un particular interés para la geografía aplicada, ya que esa condición permite una planificación preventiva, definiendo así a la ZC de la Región del Maule como un espacio aún planificable.

1 Proyecto FONDECYT No 1060745 . Artículo recibido el 19 de junio de 2009 y aceptado el 27 de julio de 2009.
Los actuales enfoques de la planificación de la ZC la consideran una entidad espacial con características propias, vinculadas a la interacción de procesos marinos, terrestres y atmosféricos, siendo además sometida a una creciente intervención antrópica (Cendrero, 1989; Charlier, 1989). Las características antes señaladas generan una paradoja en la ZC: por una parte, es un espacio frágil y vulnerable desde el punto de vista ambiental, debido a que corresponde a una interfase entre atmósfera, hidrósfera y litósfera en la cual existen condiciones de equilibrio dinámico

\footnotetext{
2 Instituto de Geografía, Pontificia Universidad Católica de Chile (Chile). E-mail: bandrade@uc.cl; farenasv@uc.cl; mlagoslo@uc.cl
} 
fácilmente alterables y, por otra, es también un territorio particularmente atrayente para el asentamiento humano. Por lo anterior, este espacio requiere de enfoques particulares de planificación, en los cuales las variables que involucran al medio natural deben ser tomadas en cuenta con una marcada atención.

Entre las múltiples variables del medio natural que conviene incluir en las actividades de planificación física del territorio, una de las más adecuadas es la geomorfológica, dado el carácter sintético de esta disciplina, en particular porque se asume que el estado de morfoconservación de las geoformas no solo refleja la evolución natural del medio, sino, también, es una expresión de cuán acertada ha sido la toma de decisiones respecto de las modalidades de ocupación del espacio.

Otro de los aspectos fundamentales para la planificación física de la ZC chilena, es la permanente amenaza de tsunami, propia de un área de interfase marina localizada sobre un margen convergente de placas. Particularmente, se debe destacar que la ZC centro-sur de la Región del Maule se localiza en la calma sísmica más antigua de Chile. El último terremoto interplaca generador de un destructivo tsunami ocurrió el 20 de febrero de 1835. Han transcurrido 174 años desde este evento y la probabilidad de que ocurra un evento similar aumenta cada día.

Figura $\mathrm{N}^{\circ} 1$

Área de estudio

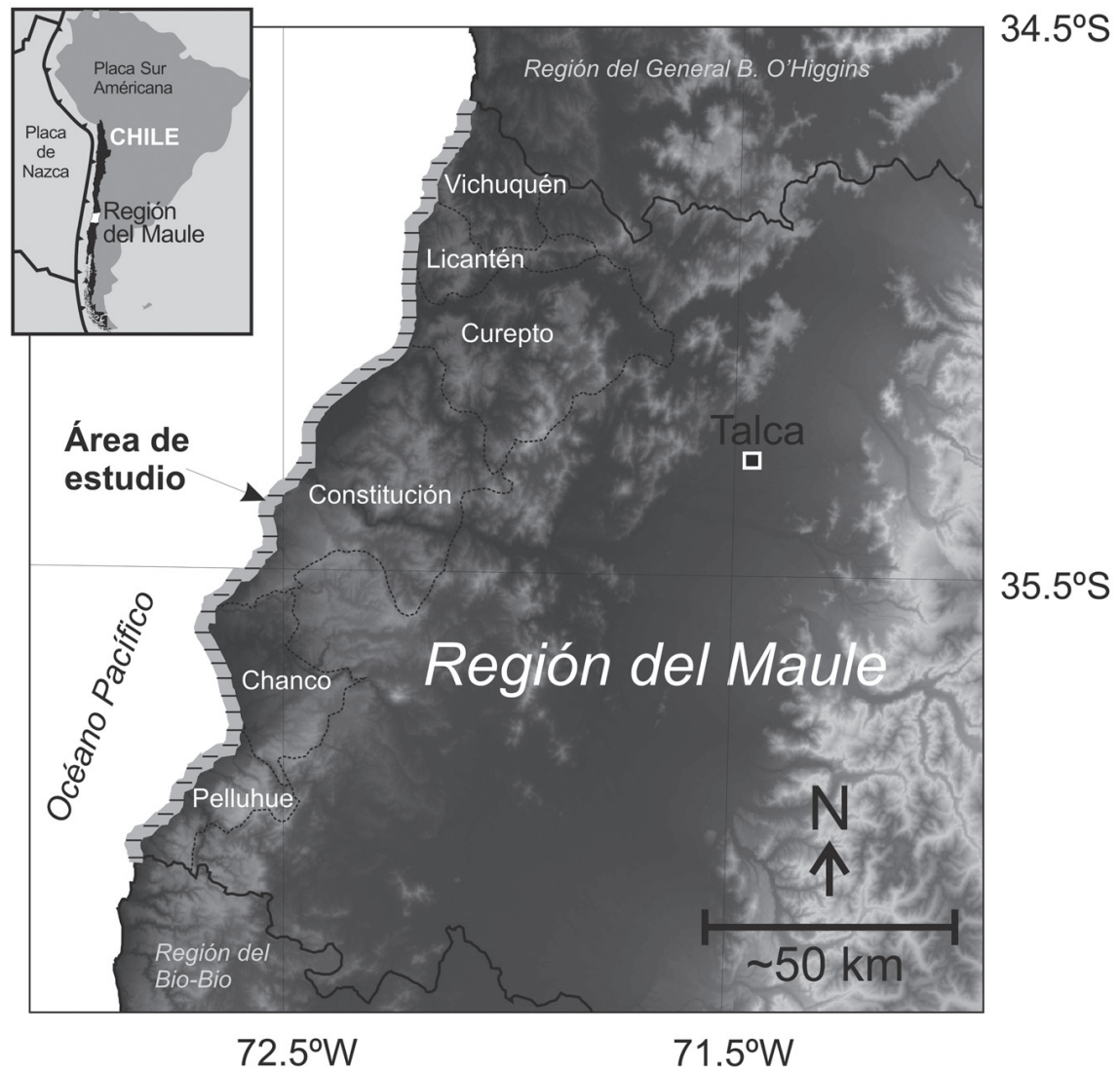

Fuente: Elaboración propia sobre modelo digital de elevación SRTM (Farr et al., 2007). 


\section{Metodología}

Para establecer una jerarquización del grado de fragilidad ambiental de diferentes unidades naturales en el área de estudio, primero se identificaron dichas unidades bajo una óptica geomorfológica, elaborando una cartografía a escala detallada desde el punto de vista de su génesis, combinando el método de la carta geomorfológica de Francia (Joly \& Tricart, 1971) y el sistema de cartografía geomorfológica del ITC (Verstappen \& Van Zuidam, 1968), mediante un levantamiento detallado de terreno y la interpretación de información proporcionada por diversos sensores remotos.

La jerarquización del grado de fragilidad ambiental de las unidades definidas en el paso anterior, consistió en precisar el área afectada por los procesos morfogenéticos actuales para cada una de estas unidades, entendiendo estos procesos como aquellos que involucran la movilización de partículas por diversos agentes de erosión (erosión en el sentido amplio del término, esto es, erosión, transporte y depositación de partículas).

Los procesos morfogenéticos actuales identificados fueron los de carácter eólico, que involucran a la deflación, reptación y acumulación; los de escurrimiento superficial elemental de los interfluvios, definidos mediante indicadores morfológicos observados en terreno y análisis de sensores remotos (descabezamiento de suelos, regueras, cárcavas y badlands); los de carácter fluvial que involucran el desbaste, la saltación, reptación y acumulación ligada a la acción hidráulica $y$, finalmente, las acciones marinas litorales responsables de la movilización de sedimentos en las playas y estuarios.

El criterio principal para evaluar la fragilidad ambiental de las unidades (estado de morfoconservación) fue el utilizado por Andrade et al. (2004), consistente en la estimación del porcentaje de superficie afectada por los procesos morfogenéticos actuales identificados. De esta manera se consideró que la fragilidad ambiental de las unidades fisiográficas es directamente proporcional a la importancia areal de los fenómenos morfogenéticos actuales desarrollados en ellas. El concepto "actual" involucra a aquellos procesos morfogenéticos desarrollados en tiempos históricos, por lo tanto, las áreas recientemente intervenidas (por ejemplo, forestación) son consideradas de acuerdo a la dinámica que presentaban antes de las modificaciones antrópicas que hoy presentan. El resultado de esta determinación de fragilidad de unidades espaciales entregó como producto tres tipos de cartas: carta de unidades fisiográficas, carta de procesos actuales y carta de fragilidad, todas ellas fueron elaboradas a escala 1:50.000.

El otro criterio a incluir para perfeccionar las herramientas de planificación física de la ZC, es el fenómeno tsunami. Para evaluar el peligro de tsunami, se reconstruyó el último evento significativo en el área de estudio, correspondiente al del 20 de febrero de 1835. Para ello se utilizó una técnica de simulación numérica basada en el modelo no-lineal TUNAMI-N2. El modelo consta de las ecuaciones de movimiento no lineales para aguas someras, integradas verticalmente y la ecuación de continuidad, sin el término de efecto Coriolis (Goto et al., 1997). La condición inicial del tsunami se determinó utilizando el modelo propuesto por Mansinha y Smylie (1971), el cual supone una deformación instantánea de la superficie del océano idéntica a la deformación vertical del fondo marino. Para la modelación del terremoto tsunamigénico se utilizaron los parámetros macrosísmicos propuestos por SHOA (2002), basados en los trabajos de Bart y Ruff (1991) y Kausel y Ramírez (1992) (Cuadro $\mathrm{N}^{\circ} 1$ ).

La propagación del tsunami se simuló utilizando el método de Goto y Ogawa (1992), que consiste en la integración numérica de las ecuaciones de aguas someras utilizando el método de diferencias finitas. La inundación de las zonas costeras se determinó empleando la condición de frontera móvil propuesta por Iwasaky y Mano (1979). La ecuación de continuidad y las ecuaciones de movimiento se discretizan en un esquema a diferencias finitas centrales denominado "salto de rana". El dominio de integración se compone de un conjunto de grillas anidadas, siendo las de mayor resolución espacial las cercanas a la costa $(90 \mathrm{~m})$. 
El dominio de integración se elaboró combinando información batimétrica y topográfica en formato digital. Con una longitud aproximada de $800 \mathrm{~km}$ desde Quintay por el norte $\left(33,21^{\circ} \mathrm{S}\right)$ hasta Bahía Mansa por el sur $\left(40,57^{\circ} \mathrm{S}\right)$ y un ancho aproximado de $425 \mathrm{~km}$ desde el océano Pacífico $\left(75,92^{\circ} \mathrm{W}\right)$ hasta el interior del continente $\left(71,12^{\circ} \mathrm{W}\right.$ ) (Figura $\mathrm{N}^{\circ}$ 3). Los datos batimétricos integran el levantamiento realizado por Smith y Sandwell (1997) y cartas náuticas del Servicio Hidrográfico y Oceanográfico de la Armada de Chile (SHOA). La topografía del área de estudio integró el levantamiento del Shuttle Radar Topography Mission - SRTM (Farr et al., 2007) y mapas topográficos del Instituto Geográfico Militar (IGM) escala 1:50.000. El resultado es la generación de un modelo digital de elevación (MDE) de topografía y batimetría de alta resolución espacial.

La simulación numérica se realizó durante cuatro horas, determinándose las alturas máximas de tsunami en las costas de la Región del Maule. Los resultados de la modelación fueron validados con datos observados de las consecuencias del terremoto y tsunami de 1835, recopilados por Charles Darwin y Robert Fitz-Roy (Fitz-Roy, 1839).

La información sobre las condiciones naturales, precisadas mediante los pasos metodológicos señalados, se utilizó como insumo para evaluar si estos aspectos se incluyen en los instrumentos de planificación territorial (IPT) vigentes. Esto se resolvió bajo una óptica espacial, integrando las capas temáticas gestionadas en un Sistema de Información Geográfica; toda la información fue georreferenciada al elipsoide y datum WGS 1984. Lo anterior permitió contrastar las coincidencias entre las asignaciones de uso determinadas por las distintas herramientas normativas y las aptitudes naturales del espacio costero.

Finalmente, se propuso, en un área específica del espacio estudiado, una zonificación con fines de ordenamiento territorial, específicamente en una parte del borde costero de las comunas de Chanco y Pelluhue, dado que este es el único tramo de la costa estudiada que cuenta con un instrumento de ordenamiento territorial vigente actualizado.

\section{Resultados y discusión}

\section{Fragilidad ambiental}

El levantamiento de la cartografía de unidades fisiográficas escala 1:50.000 permitió identificar diez unidades naturales principales que dan cuenta de la constitución natural del área de estudio y la importancia de la superficie correspondiente de cada unidad (Cuadro $\mathrm{N}^{\circ}$ 2).

El Cuadro $N^{\circ} 2$ muestra un claro predominio de las unidades núcleo montañoso, con más de $197 \mathrm{~km}^{2}$, seguido por la unidad terraza marina, con más de $192 \mathrm{~km}^{2}$ y por la unidad duna actual, con $160 \mathrm{~km}^{2}$. El conjunto de las otras unidades presenta una proporción de superficie individual inferior al 50\% de esta última.

Las unidades más activas, desde el punto de vista morfogenético, son aquellas que presentan una mayor superficie afectada por la movilización de partículas (Cuadro $N^{\circ} 2$ ). En el área de estudio las unidades más frágiles,

Cuadro $\mathrm{N}^{\circ} 1$

Parámetros de falla utilizados para simular el tsunami de 1835

\begin{tabular}{|c|c|c|c|c|c|c|c|c|}
\hline $\begin{array}{c}\text { Longitud, } \\
L \\
(\mathrm{~km})\end{array}$ & $\begin{array}{c}\text { Ancho, } \\
W \\
(\mathrm{~km})\end{array}$ & $\begin{array}{c}\text { Deslizamiento, } \\
(\mathrm{m})\end{array}$ & $\begin{array}{c}\text { Rumbo, } \\
(\text { grados })\end{array}$ & $\begin{array}{c}\text { Echado, } \\
(\text { grados })\end{array}$ & $\begin{array}{c}\text { Ángulo, } \\
(\text { grados })\end{array}$ & $\begin{array}{c}\text { Profundidad, } \\
\begin{array}{c}H \\
(\mathrm{~km})\end{array}\end{array}$ & $\begin{array}{c}\text { Lat* } \\
(\mathrm{S})\end{array}$ & $\begin{array}{c}\text { Lon* } \\
(\mathrm{W})\end{array}$ \\
\hline 420 & 120 & 10 & 30 & 20 & 90 & 10 & 37,9833 & 74,4094 \\
\hline
\end{tabular}

* Coordenada de esquina SW.

Fuente: SHOA, 2002. 
es decir, las morfogenéticamente más activas, corresponden a las playas, en las que el $100 \%$ de su superficie presenta movilización de partículas y ellas representan el $2 \%$ de la superficie del área de estudio. Igualmente, el $100 \%$ de las áreas ocupadas por los lechos fluviales (equivalente al $2 \%$ del área de estudio) muestran una activa movilización de partículas.

El $100 \%$ de la superficie ocupada por la unidad duna actual, sean estas dunas libres o forestadas, están sujetas real o potencialmente a movilización de sedimentos por el viento. Esta unidad representa el $21,7 \%$ de la superficie total del área de estudio. La unidad estuarios y salinas, que corresponde al $0,2 \%$ del área de estudio, también presenta el $100 \%$ de su superficie con procesos comandados por las mareas y corrientes asociadas (Figura $\mathrm{N}^{\circ} 2$ ).

Lo anterior ha llevado a considerar que las unidades arriba señaladas, equivalentes a un cuarto de la superficie del área de estudio, presentan un grado de fragilidad extrema.
Desde el punto de vista de su magnitud en superficie, los procesos ligados al escurrimiento superficial elemental de los interfluvios afectan en porcentaje variable al resto de las unidades fisiográficas. Se ha considerado que las unidades núcleo montañoso, acantilado y terrazas marinas presentan una fragilidad alta, con valores que oscilan entre un $13,29 \%$ y un $10,15 \%$ de su superficie afectada por procesos actuales. Las laderas de quebrada, que presentan un $6,4 \%$ de su superficie sometida a procesos actuales, han sido categorizadas como áreas con fragilidad media. Finalmente, la unidad terraza fluvial muestra una baja fragilidad con respecto a los procesos actuales, con menos de $1 \%$ de su superficie afectada. De acuerdo a estos resultados obtenidos, se clasificó las unidades fisiográficas en cuatro categorías de fragilidad ambiental (Cuadro $N^{\circ} 3$ ).

Las categorías de fragilidad se generaron al comparar entre sí las unidades desde el punto de vista de su grado de morfoconservación, cuantificando las superficies afectadas por procesos actuales en cada una de ellas.

Cuadro $\mathrm{N}^{\circ} 2$

Unidades fisiográficas, superficie y procesos actuales

\begin{tabular}{|c|c|c|c|c|}
\hline $\begin{array}{l}\text { Tipo de } \\
\text { procesos }\end{array}$ & Unidades & $\begin{array}{l}\text { Área total } \\
\quad\left(\mathrm{km}^{2}\right)\end{array}$ & $\begin{array}{l}\text { Área con procesos } \\
\text { actuales }\left(\mathrm{km}^{2}\right)\end{array}$ & $\begin{array}{c}\text { Procesos } \\
\text { actuales (\%) }\end{array}$ \\
\hline $\begin{array}{l}\text { Escurrimiento } \\
\text { superficial } \\
\text { elemental en } \\
\text { interfluvio }\end{array}$ & $\begin{array}{l}\text { Ladera de acantilado } \\
\text { Ladera de quebrada } \\
\text { Núcleo montañoso } \\
\text { Terraza fluvial } \\
\text { Terraza marina } \\
\text { Terraza marina baja }\end{array}$ & $\begin{array}{r}15,83 \\
70,42 \\
197,25 \\
32,10 \\
192,91 \\
10,07\end{array}$ & $\begin{array}{r}2,06 \\
4,48 \\
26,21 \\
0,22 \\
16,16 \\
0,18\end{array}$ & $\begin{array}{r}12,98 \\
6,37 \\
13,29 \\
0,68 \\
8,38 \\
1,77\end{array}$ \\
\hline Eólicos & Duna & 160,89 & 160,89 & 100,00 \\
\hline Fluviales & Lecho fluvial & 15,46 & 15,46 & 100,00 \\
\hline Marinos & $\begin{array}{l}\text { Playa } \\
\text { Estuarios y salinas }\end{array}$ & $\begin{array}{l}14,81 \\
22,38\end{array}$ & $\begin{array}{l}14,81 \\
22,38\end{array}$ & $\begin{array}{l}100,00 \\
100,00\end{array}$ \\
\hline Excluida & $\begin{array}{l}\text { Duna indeterminada } \\
\text { Lago } \\
\text { Plano lacustre } \\
\text { Zona urbana } \\
\text { Total }\end{array}$ & $\begin{array}{r}0,281 \\
2,715 \\
0,622 \\
5,282 \\
741,03\end{array}$ & $\begin{array}{c}- \\
- \\
- \\
- \\
262,85\end{array}$ & $\begin{array}{c}- \\
- \\
- \\
- \\
35,47\end{array}$ \\
\hline
\end{tabular}

Fuente: Elaboración propia. 


\section{Figura $\mathrm{N}^{\circ} 2$}

Duna activa en Putú-Quivolgo: la movilización generalizada de partículas se manifiesta por el avance de su frente sobre el camino, sepultando parcialmente renovales

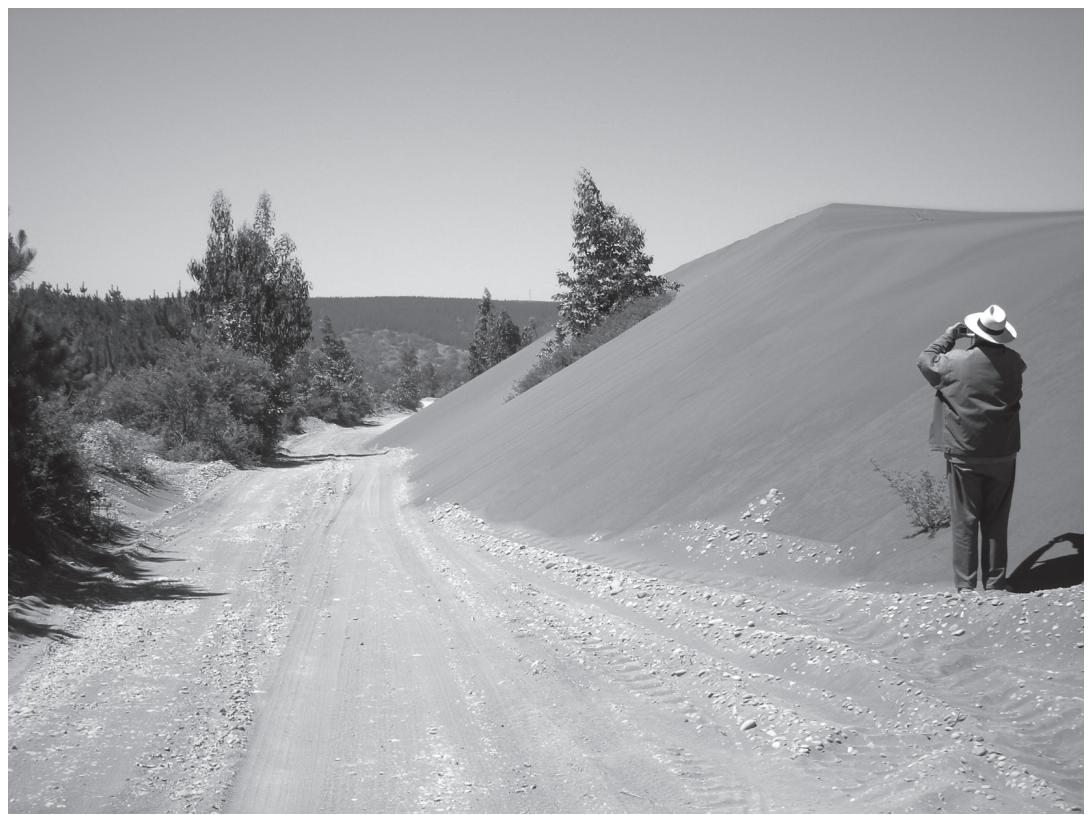

Fuente: Colección personal de los autores, 2008.

\section{Cuadro $\mathrm{N}^{\circ} 3$}

Grados de fragilidad ambiental para las unidades fisiográficas

\begin{tabular}{|c|c|}
\hline Grado de fragilidad & Descripción \\
\hline Extrema & $\begin{array}{l}\text { En el área de estudio, cuando el } 100 \% \text { de una unidad natural presenta } \\
\text { movilización de partículas, corresponde a playas, lechos fluviales, duna } \\
\text { actual, estuarios y salinas (véase Figura } N^{\circ} 2 \text { ) }\end{array}$ \\
\hline Alta & $\begin{array}{l}\text { En el área de estudio, corresponde a las unidades núcleo montañoso, } \\
\text { acantilado y terrazas marinas. Unidades donde los procesos actuales se } \\
\text { manifiestan entre un } 10,15 \% \text { y } 13,29 \% \text { de su superficie }\end{array}$ \\
\hline Media & $\begin{array}{l}\text { En el área de estudio, corresponde a la unidad natural laderas de que- } \\
\text { brada, donde un } 6,4 \% \text { de su superficie se encuentra expuesta a procesos } \\
\text { actuales }\end{array}$ \\
\hline Baja & $\begin{array}{l}\text { En el área de estudio, corresponde a la unidad terraza fluvial, la que pre- } \\
\text { senta procesos actuales inferiores al } 1 \% \text { de su superficie }\end{array}$ \\
\hline
\end{tabular}

Fuente: Elaboración propia. 
Por lo anterior, la fragilidad de las unidades puede variar de un contexto morfoclimático a otro independientemente de su génesis $y$, en consecuencia, las categorías de fragilidad definidas en esta investigación solo son válidas para esta área de estudio. Refuerza esta idea de definir categorías de fragilidad mediante la comparación entre las unidades del área de estudio, el hecho que una parte importante de los procesos actuales ha sido desencadenada por diferentes interferencias antrópicas, relacionadas con diversas actividades productivas cuya intensidad varía de acuerdo a la expresión espacial de los modos o géneros de vida, en el sentido del concepto planteado por Vidal de la Blache (1911). En consecuencia, las clases de fragilidad definidas corresponden a categorías de valoración relativa.
Las condiciones naturales del espacio costero son el resultado de procesos morfogenéticos actuantes sobre unidades fisiográficas variadas, las que igualmente presentan diferentes intensidades de intervención antrópica; por lo tanto, las respuestas evolutivas del medio son diferentes bajo distintos contextos morfoclimáticos y son propias de cada territorio. Por lo anterior, las categorías de fragilidad definidas en esta investigación solo son válidas para este caso de estudio.

\section{Peligro de tsunami}

Los resultados de la simulación numérica muestran las máximas alturas de ola registradas en la línea de costa (Figura $N^{\circ} 3$ ). Se observa cómo las mayores amplitudes se

Figura $N^{\circ} 3$

Dominio de integración y alturas máximas de tsunami en la costa para el evento de 1835

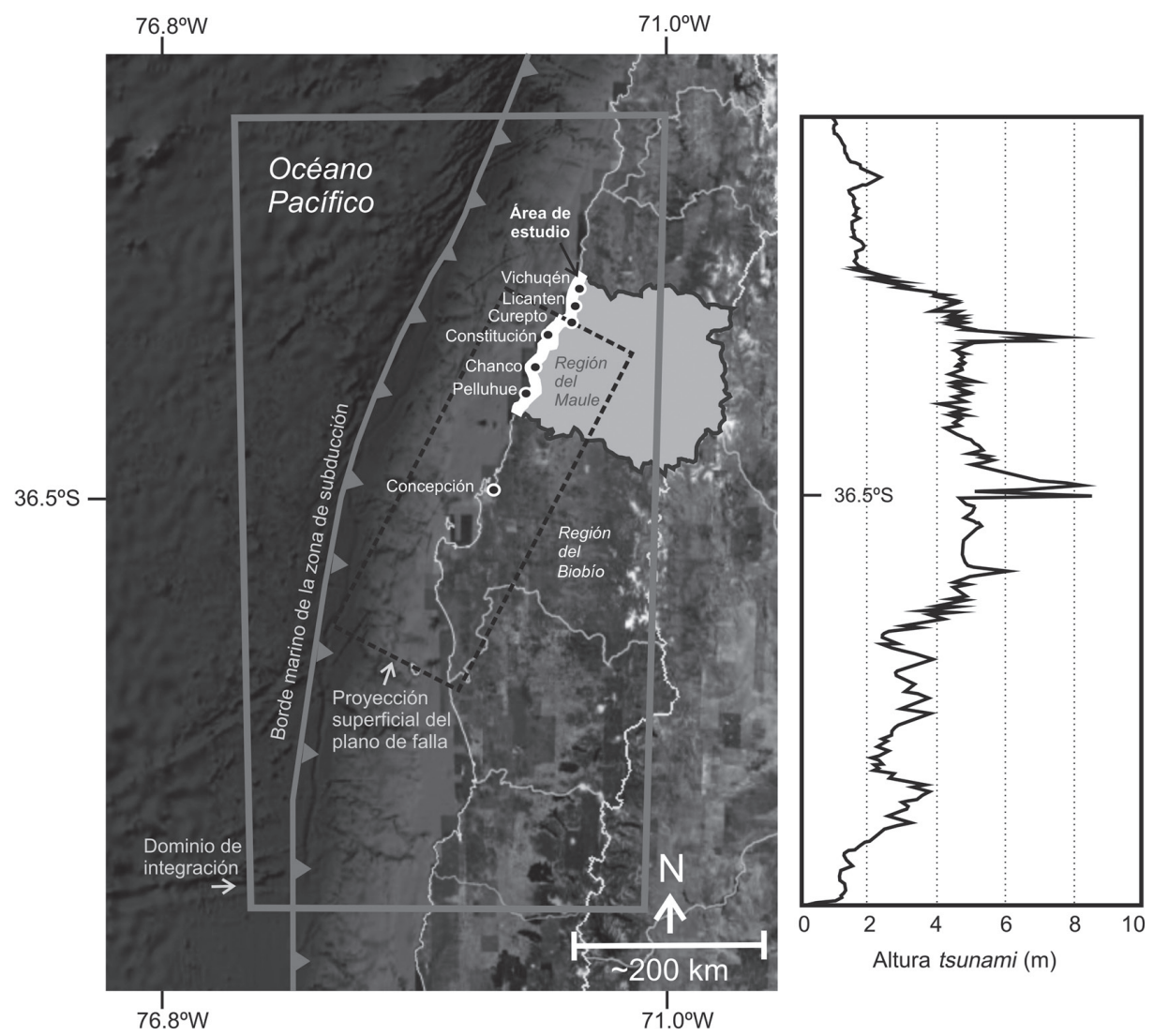

Fuente: Elaboración propia sobre mosaico de imágenes y fondo marino de Google Earth. 
concentran en la zona de ruptura afectando las costas de las regiones de Maule y Biobío. Se observa que las máximas alturas de ola alcanzan cerca de $8 \mathrm{~m}$, concentrándose en la zona costera de Constitución y la bahía de Concepción. Este valor coincide con lo observado por Darwin en esta última, quien indica que las aguas se elevaron a una altura vertical de 23 pies por encima del nivel de las más altas mareas ( 7-8 $\mathrm{m})$.

Considerando las alturas de ola máximas modeladas para el área de estudio, amplitudes que alcanzaron casi los $8 \mathrm{~m}$, más el rango de las mareas de sicigia de 1,66 m (SHOA, 2008), se definió como cota máxima de inundación la altitud $10 \mathrm{~m}$. En este escenario, todas las áreas pobladas de la Región del Maule localizadas bajo esta altitud se encontrarían bajo riesgo.

\section{Los Instrumentos de Planificación Territorial (IPT) vigentes}

Se analizó la situación de la planificación territorial en la Región del Maule y en particular en su zona costera (Cuadro $\mathrm{N}^{\circ}$ 4). En este análisis se examinó si las consideraciones ambientales y de riesgo estaban incluidas en dichos instrumentos (Andrade et al., 2008).

El análisis de los IPT vigentes indica que la situación de la planificación territorial en la ZC en la Región del Maule no es satisfactoria, dado que la región no cuenta con un instrumento de escala regional, y en cuatro de las seis comunas costeras (Licantén, Curepto, Chanco y Pelluhue) no existe un Plan Regulador Comunal (PRC) vigente. La regulación es muy débil ya que se reduce solo a la existencia de límites urbanos en algunos pueblos de dichas comunas (Chanco, Curepto, Iloca, Curanipe, Pelluhue), los que, dada su antigüedad, han sido completamente superados por el crecimiento de dichos centros. En las cuatro comunas los PRC se encuentran en formulación y por lo tanto no están vigentes (Andrade et al., 2008).

Solo en dos comunas existe un PRC vigente (Constitución desde 1988 y Vichuquén desde 1967), aunque en ambos casos contienen una regulación bastante básica y no bien delimitada para los requerimientos actuales de dichos centros urbanos, por lo que están en proceso de actualización. En consecuencia, la Región del Maule puede considerarse, hasta el momento del cierre del proyecto de investigación que da origen a este artículo, entre las más rezagadas del país en materia de regulación urbana (Guijón, 2007). Lo anterior, a pesar de la existencia de un Plan Regulador Intercomunal que incluye las comunas de Cauquenes, Chanco y Pelluhue y que incorpora algunas consideraciones de carácter ambiental.

A partir de las modificaciones impulsadas en Chile durante 2009 en materia de descentralización, desde la Subsecretaría de Desarrollo Regional y Administrativo (SUBDERE), los Planes Regionales de Desarrollo Urbano (de responsabilidad del Ministerio de Vivienda y Urbanismo) serán sustituidos por los nuevos Planes Regionales de Ordenamiento Territorial (PROT), de responsabilidad de los Gobiernos Regionales (GORE). Los GORE asumirán, además, la responsabilidad de la planificación regional que hasta ahora estaba en manos del Ministerio de Planificación, a través de las Secretarías Regionales Ministeriales de Planificación (SERPLAC).

Las modificaciones señaladas permitirán mirar el espacio regional de manera integra$\mathrm{da}$, superando las restricciones que impone el enfoque parcializado de la mirada sectorial y probablemente facilitando, en el futuro, la incorporación de variables ambientales en los IPT.

Del análisis de los resultados de la investigación, respecto de los niveles de fragilidad existentes en la zona costera regional (Cuadro $\mathrm{N}^{\circ} 5$ ), se desprende que el $40,7 \%$ de la superficie regulada en la zona costera por los instrumentos vigentes, presenta una fragilidad alta, porcentaje que se eleva a un $47,3 \%$ si se suma la superficie con fragilidad extrema $(6,7 \%)$.

El Cuadro $N^{\circ} 5$ permite observar la relación entre superficie por tipo de fragilidad y los límites urbanos de las localidades de Chanco, Curanipe y Pelluhue, y con el límite urbano del PRC de Constitución del año 1988. Como se puede apreciar, en el caso de Chanco, alrededor del $21 \%$ de la superficie $(15,1 \mathrm{ha})$, incluida en el límite urbano esta- 


\section{Cuadro $\mathrm{N}^{\circ} 4$}

Instrumentos de Planificación Territorial (IPT) vigentes para la zona costera de la Región del Maule

\begin{tabular}{|c|c|c|c|}
\hline Instrumento & \multicolumn{2}{|c|}{ Situación actual } & Incorporación de fragilidad ambiental y riesgo \\
\hline PRDU & \multirow{2}{*}{\multicolumn{2}{|c|}{$\begin{array}{l}\text { No hay } \\
\text { PRI Cauquenes-Chanco-Pelluhue, } 2003\end{array}$}} & - \\
\hline PRI & & & $\begin{array}{l}\text { Norma construcción en zonas de pendiente, } \\
\text { mandata a los nuevos PRC para incorporar } \\
\text { restricciones específicas para acantilados o } \\
\text { talud del litoral, terrenos de valor paisajístico, } \\
\text { ambiental y ecológico, de conservación, de } \\
\text { riesgo por eventos naturales, zonas de protec- } \\
\text { ción costera (playas de mar con faja no edi- } \\
\text { ficable de } 20 \text { m de ancho mínimo), acceso a } \\
\text { playas y caletas pesqueras, cauces naturales, } \\
\text { entre otros. }\end{array}$ \\
\hline \multirow[t]{6}{*}{ PRC } & Comuna de Chanco & No hay & - \\
\hline & $\begin{array}{l}\text { Comuna de } \\
\text { Constitución }\end{array}$ & $\begin{array}{l}\text { D. S. } 189,1987, \text { MINVU, D. O. } \\
06.02 .1988 \\
\text { D. S. } 60,1990, \text { MINVU, D. O. } \\
\text { 15.06.1991 (modificación PRC) }\end{array}$ & $\begin{array}{l}\text { Incorpora algunas restricciones en bienes } \\
\text { nacionales de uso público, tales como vías, } \\
\text { playas y áreas verdes y en áreas especiales, } \\
\text { tales como márgenes de río y orillas de mar, } \\
\text { grandes parques, zonas de protección ecoló- } \\
\text { gica, quebradas, pantanos, etc. }\end{array}$ \\
\hline & Comuna de Curepto & No hay & - \\
\hline & Comuna de Licantén & $\begin{array}{l}\text { D. S. } 3.508,1951, \text { MOP, D. O. } \\
08.10 .1951\end{array}$ & No hay incorporación \\
\hline & Comuna de Pelluhue & No hay & - \\
\hline & $\begin{array}{l}\text { Comuna de } \\
\text { Vichuquén }\end{array}$ & $\begin{array}{l}\text { D. S. } 452,1967, \text { MINVU, D. O. } \\
08.09 .1967\end{array}$ & No hay incorporación \\
\hline $\begin{array}{l}\text { Planos } \\
\text { seccionales }\end{array}$ & \multicolumn{2}{|c|}{$\begin{array}{l}\text { Comuna de Vichuquén: Modificación PRC según Plano } \\
\text { Seccional La Isla No } 1 \text { (D. S. 15, 1987, MINVU, D. O. } \\
\text { 27.02.1987). }\end{array}$} & No hay incorporación \\
\hline \multirow[t]{6}{*}{ Límite urbano } & Comuna de Chanco & $\begin{array}{l}\text { D. S. } 311,1969, \text { MINVU, D. O. } \\
\text { 30.04.1969 }\end{array}$ & No hay incorporación \\
\hline & $\begin{array}{l}\text { Comuna de } \\
\text { Constitución }\end{array}$ & No hay & - \\
\hline & Comuna de Curepto & $\begin{array}{l}\text { D. S. } 750,1968, \text { MINVU, D. O. } \\
06.11 .1968\end{array}$ & No hay incorporación \\
\hline & Comuna de Licantén & No hay & - \\
\hline & Comuna de Pelluhue & $\begin{array}{l}\text { Curanipe (D. S. } 69,1970, \\
\text { MINVU, D. O. 18.02.1970, } \\
\text { antigua comuna de Chanco) } \\
\text { Pelluhue (D. S. } 70,1970, \\
\text { MINVU, D. O. 18.02.1970) }\end{array}$ & No hay incorporación \\
\hline & $\begin{array}{l}\text { Comuna de } \\
\text { Vichuquén }\end{array}$ & $\begin{array}{l}\text { Mal Paso y Santa Rosa (D. S. 79, } \\
\text { 1969, MINVU, 12.02.1969) } \\
\text { Vichuquén, Llico, Piedras } \\
\text { Bayas, Quesería y La Isla (D. } \\
\text { S. 55, 1972, MINVU, D. O. } \\
\text { 12.02.1972) }\end{array}$ & No hay incorporación \\
\hline
\end{tabular}

Fuente: Modificado de Guijón, 2007. 
blecido en 1969, corresponde a un área considerada de fragilidad extrema, de acuerdo a los resultados del proyecto. Si se adiciona la categoría de fragilidad alta, un $96 \%$ de la superficie definida por el límite urbano queda incluida en áreas ambientalmente inadecuadas.

Esta situación es similar a los resultados obtenidos en los casos de Curanipe y Pelluhue, si se considera ambas categorías de fragilidad (alta y extrema), alcanzando valores de $91,5 \%$ y $94,26 \%$, respectivamente.

En el caso del límite urbano definido en el PRC de Constitución, cerca de un tercio de la superficie urbana allí definida corresponde, según nuestros resultados, a la categoría de fragilidad extrema y alta.

Como se indicó anteriormente, existe un Plan Regulador Intercomunal (PRI) que incluye dos de las comunas costeras (Chanco y Pelluhue), además de la comuna interior de Cauquenes, el que si bien tiene importancia en términos de uso del borde costero, al no estar enfocado a un desarrollo particular de dicho borde y emplear los mismos criterios para las tres comunas consideradas, no recoge la marcada diversidad espacial propia del borde costero. Adicionalmente, este instrumento está en fase de revisión para introducirle algunas modificaciones, aunque estas no están aún vigentes.
En el análisis detallado realizado por Guijón (2007), se ha constatado que a pesar del esfuerzo que la Secretaría Regional Ministerial de Vivienda y Urbanismo para que todas las comunas de la región cuenten con su respectivo PRC, aquellos de la zona costera encuentran dificultades para su aprobación.

Se ha constatado que la actual situación de antigüedad y vigencia de los instrumentos de ordenamiento territorial en el área de estudio, mantiene ambientalmente desprotegida a la zona costera, frente a la creciente demanda de uso del suelo, similar a otras áreas litorales del país. Un ejemplo del tipo de problema al cual se enfrentan las comunas costeras de la región es el caso de la comuna de Licantén, la que solo cuenta con una zona urbana, en el balneario de lloca, cuyo límite urbano data de 1951. Las descripciones de los límites urbanos son topológicas y por lo tanto de escasa precisión; el decreto que fija la zona urbana entrega como límite occidental de esta simplemente "a la playa del balneario". Adicionalmente, en lo que se refiere a lloca, el proyecto de nuevo plan regulador no ha podido ser definido para su presentación ante la Comisión Regional de Medio Ambiente ya que todavía está en duda el trazado que seguirá la carretera longitudinal costera en esta zona. Una decisión tan importante para el desarrollo urbano de una localidad, como tantas veces, tendrá un marcado carácter alotópico.

\section{Cuadro $\mathrm{N}^{\circ} 5$}

Superficie por tipo de fragilidad, regulada por límites urbanos (ha)

\begin{tabular}{|l|c|c|c|c|}
\hline \multirow{2}{*}{ Fragilidad } & \multicolumn{4}{|c|}{ Comunas } \\
\cline { 2 - 5 } & Chanco & Constitución & Curanipe & Pelluhue \\
\hline Baja & 0,0 & 0,0 & 2,3 & 5,8 \\
Media & 2,6 & 3,8 & 1,5 & 0,5 \\
Alta & 53,6 & 241,7 & 28,5 & 83,3 \\
Extrema & 15,1 & 24,1 & 12,6 & 14,9 \\
Sin información & 0,0 & 510,8 & 0,0 & 0,0 \\
Superficie límite urbano & 71,2 & 780,4 & 44,9 & 104,6 \\
\hline
\end{tabular}

Fuente: Elaboración propia. 
Las comunas costeras de la región cuentan con algunas áreas silvestres protegidas (dos reservas nacionales y dos santuarios de la naturaleza) y algunos monumentos nacionales, además de un plan regional de desarrollo turístico. Sin embargo, en lo relativo al borde costero, la Región del Maule no cuenta con una zonificación para su litoral. De acuerdo con lo indicado en la Oficina del Borde Costero, recién se empieza a hablar de un estudio de zonificación costera.

Respecto de la incorporación de la variable fragilidad ambiental en los instrumentos de planificación territorial vigentes, en el caso del PRI Cauquenes-Chanco-Pelluhue, si bien se incorporan restricciones específicas para la ocupación de acantilados y taludes litorales, terrenos de valor paisajístico, ambiental y ecológico o con riesgos de eventos naturales, las superficies reguladas son reducidas respecto de las áreas frágiles definidas por este proyecto. En la Figura $N^{\circ} 4$ se con- trastan las distintas zonas propuestas en el citado PRI, con la jerarquización de fragilidad ambiental definida por este estudio.

En el Cuadro $\mathrm{N}^{\circ} 6$ se puede apreciar que dentro de las áreas normadas por el PRI existen áreas definidas como de Extensión Urbana (ZEU-1 y ZEU-2) y Zona Urbana (ZU-2), que presentan un alto porcentaje de su superficie con grados de fragilidad alta y extrema. Por ejemplo en el caso de la ZU-2, el 96,9\% de su superficie corresponde a los más altos grados de fragilidad y en el caso de ZEU-2 este valor alcanza al 86,5\%; el $100 \%$ de la ZEU-1 está comprendida en un área de fragilidad alta.

También en el caso de la Zona de Desarrollo Urbano Condicionado (ZDUC), el porcentaje de fragilidad alta y extrema es elevado $(95 \%)$, aunque en este ejemplo la propia definición de la zona otorga la posibilidad de establecer condiciones o exigencias al desarrollo urbano.

Figura $\mathrm{N}^{\circ} 4$

Zonificación del PRI Chanco-Cauquenes-Pelluhue e Índice de Fragilidad Ambiental

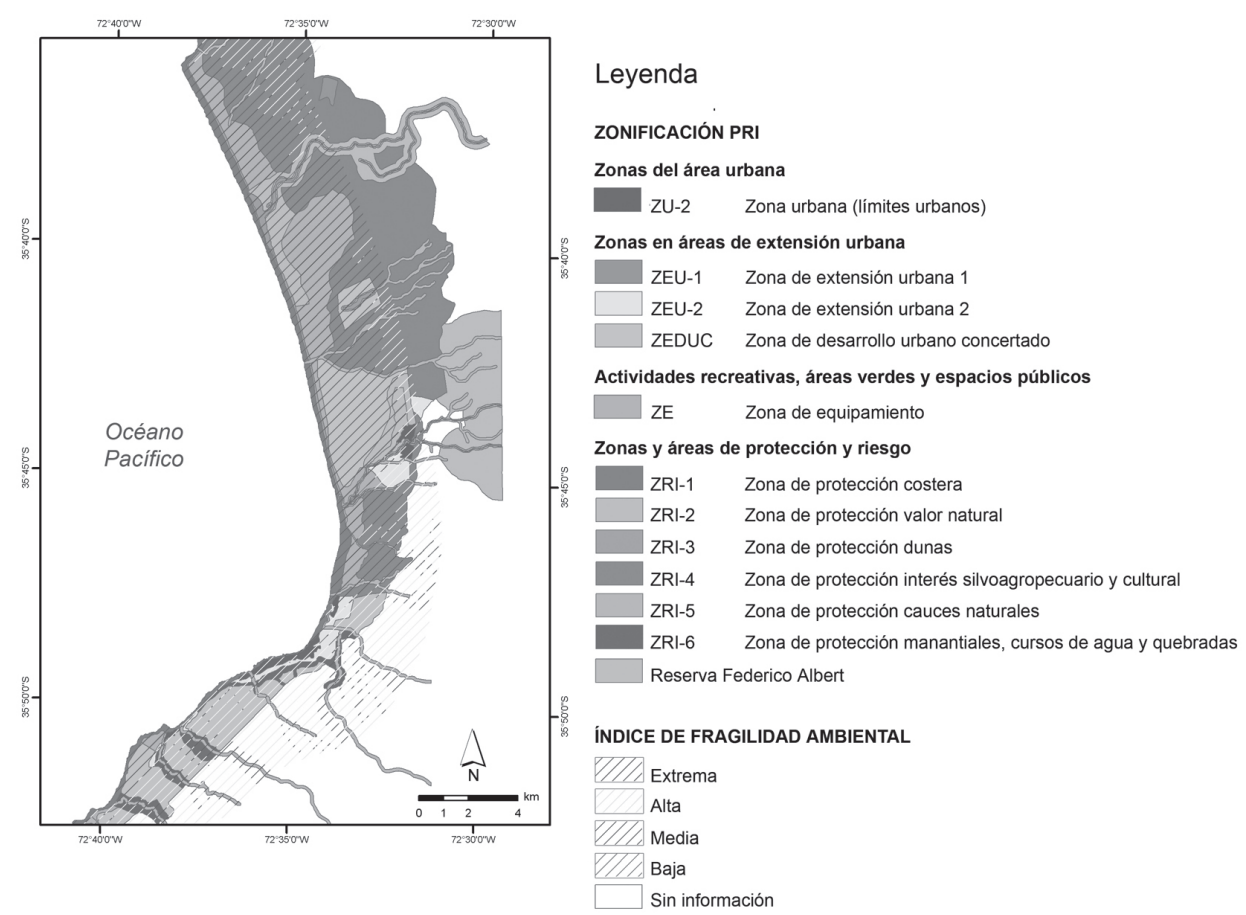

Fuente: Elaboración propia. 
Cuadro $\mathrm{N}^{\circ} 6$

Grado de fragilidad ambiental, por zonas del PRI (ha)

\begin{tabular}{|l|r|r|r|r|}
\hline \multirow{2}{*}{ Zona* } & \multicolumn{4}{|c|}{ Grado de fragilidad ambiental } \\
\cline { 2 - 5 } & Baja & Media & Alta & Extrema \\
\hline ZDUC & 7,4 & 120,3 & $1.293,4$ & $1.288,2$ \\
ZE & 38,0 & 21,5 & 116,3 & $1.933,1$ \\
ZEU-1 & 0,0 & 0,0 & 12,6 & 0,0 \\
ZEU-2 & 36,6 & 16,9 & 299,3 & 43,7 \\
ZRI-1 & 0,4 & 0,2 & 111,6 & 715,1 \\
ZRI-2 & 65,9 & 106,4 & 395,9 & 506,9 \\
ZRI-3 & 0,0 & 0,0 & 1,0 & 118,5 \\
ZRI-4 & 94,7 & 160,4 & $1.788,2$ & $2.178,9$ \\
ZRI-5 & 97,3 & 284,5 & 293,6 & 289,4 \\
ZRI-6 & 47,2 & 220,0 & 296,1 & 8,1 \\
ZU-2 & 3,4 & 2,2 & 149,0 & 30,4 \\
Sin Información & 0,0 & 1,1 & 26,8 & 1,8 \\
Total & 390,9 & 933,3 & $4.783,6$ & $7.114,2$ \\
\hline
\end{tabular}

* Ver definición de abreviaturas en Figura $N^{\circ} 4$.

Fuente: Elaboración propia.

Afortunadamente, este instrumento de planificación territorial incorpora algunas formas de restricción, tales como Protección Costera (ZRI-1), Protección de Valor Natural (ZRI-2), Protección de Amophilas (ZRI-3), Protección de Interés Silvoagropecuario y Cultural (ZRI-4) y Protección de Cauces Naturales (ZRI-5), las que incluyen áreas de fragilidad alta y extrema. Un ejemplo de ello lo constituye la ZRI-4, con un $94 \%$ de su superficie correspondiente a áreas definidas como de fragilidades altas y extremas por nuestro estudio.

Respecto de la incorporación del riesgo de tsunami en los instrumentos de planificación territorial vigentes, la revisión detallada de ellos muestra que esta variable es inexistente. No obstante esta condición, en algunas localidades costeras, tales como Duao, Constitución y Pelluhue existen puntuales señalizaciones de vías de evacuación frente a un evento de este tipo, en las que curiosamente, la flecha recomienda dirigirse hacia la ola (Figura $\mathrm{N}^{\circ} 5$ ). Esta situación permite suponer que este tipo de riesgo puede ser incorporado en futuros instrumentos de planificación territorial.

\section{Propuesta de zonificación con fines de ordenamiento territorial}

El Cuadro $N^{0} 7$ resume la propuesta de zonificación, que aunque más restrictiva que lo que se acostumbra a ver en los instrumentos de planificación territorial, apunta a poner en la discusión criterios adicionales de fragilidad y peligro de tsunami. La Figura $N^{\circ} 6$ espacializa la zonificación.

\section{Zona de máxima restricción}

En la zona de estudio, se trata de áreas que presentan el $100 \%$ de su superficie con movilización de partículas, por lo que según los resultados obtenidos, incluye playas, 
Figura $N^{\circ} 5$

Duao. Señalización rudimentaria indicando vía de evacuación a zona de seguridad en caso de tsunami

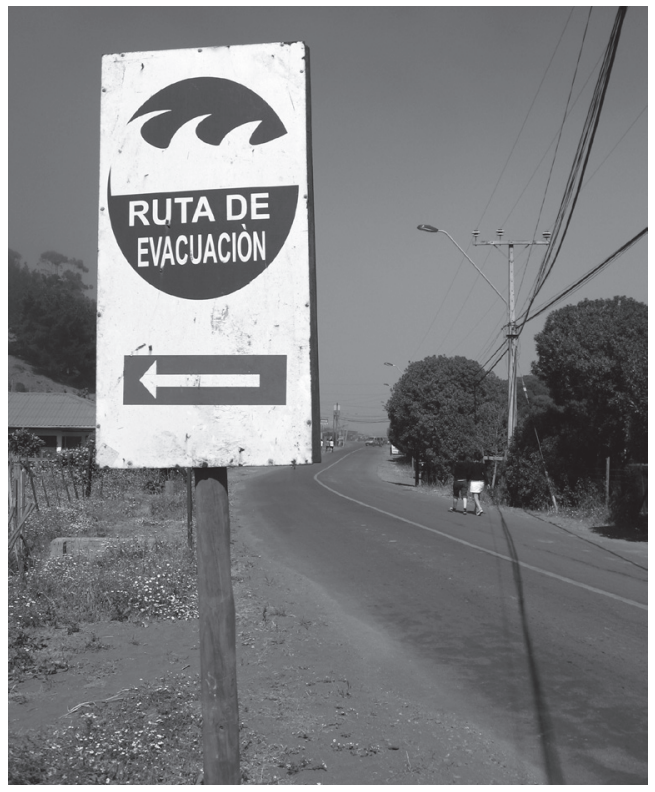

Fuente: Colección personal de los autores, 2008. lechos fluviales, duna actual, estuarios y salinas. En este caso, la recomendación de uso es: exclusión de usos. Incluye además, las áreas bajo los 10 metros de altitud, debido a su elevada exposición a la amenaza de tsunami, de manera de garantizar un manejo más sustentable del espacio costero.

\section{Zona de alta restricción}

Corresponde a áreas donde los procesos actuales se manifiestan entre un $10,15 \%$ y $13,29 \%$ de la superficie, en unidades tales como núcleo montañoso, acantilado y terrazas marinas. La recomendación de uso, en este caso es: uso restringido, con la recomendación de autorizar solo usos que no gatillen o aceleren procesos morfogenéticos, esto con el fin de conservar el carácter natural del paisaje (Figura $\mathrm{N}^{\circ} 7$ ).

\section{Zona de baja restricción}

Corresponde a áreas donde alrededor de un $6 \%$ de su superficie se encuentra expuesta a procesos actuales. En el área de estudio se refiere a la unidad natural laderas de quebra-

\section{Cuadro $\mathrm{N}^{\circ} 7$}

Propuesta de zonificación de apoyo a los instrumentos de planificación territorial

\begin{tabular}{|l|l|l|l|}
\hline \multicolumn{1}{|c|}{ Tipo de zona } & $\begin{array}{c}\text { Recomendación } \\
\text { de uso }\end{array}$ & \multicolumn{1}{c|}{ Unidades } & \multicolumn{1}{c|}{ Observaciones } \\
\hline $\begin{array}{l}\text { Máxima } \\
\text { restricción }\end{array}$ & Exclusión & $\begin{array}{l}\text { Playas, lechos } \\
\text { fluviales, duna actual, } \\
\text { estuarios y salinas }\end{array}$ & $\begin{array}{l}\text { Áreas que presentan el 100\% de su } \\
\text { superficie con movilización de par- } \\
\text { tículas } \\
\text { Áreas bajo los 10 metros de altitud } \\
\text { debido a su elevada exposición a la } \\
\text { amenaza de tsunami }\end{array}$ \\
Baja restricción & Limitado & $\begin{array}{l}\text { Núcleo montañoso, } \\
\text { acantilado y terrazas } \\
\text { marinas } \\
\text { Laderas de quebrada }\end{array}$ & $\begin{array}{l}\text { Solo usos que no gatillen o aceleren } \\
\text { procesos morfogenéticos }\end{array}$ \\
Sin restricción & $\begin{array}{l}\text { Sin mayores } \\
\text { restricciones }\end{array}$ & $\begin{array}{l}\text { Las intervenciones deben contemplar } \\
\text { medidas tendientes a evitar el des- } \\
\text { encadenamiento y/o aceleración de } \\
\text { procesos morfogenéticos }\end{array}$ \\
\hline
\end{tabular}

Fuente: Elaboración propia. 
da. La recomendación de uso es: uso limitado. Si bien las pendientes de estas unidades restringen de manera natural una ocupación antrópica intensiva, las obras civiles que pueden generarse en ellas, deben contemplar medidas tendientes a evitar el desencadenamiento y/o aceleración de procesos morfogenéticos, con el fin de conservar el carácter natural del paisaje.

\section{Zona sin restricción}

Corresponde a áreas que presentan procesos actuales inferiores al $1 \%$ de la superficie y en el área de estudio se refiere a la unidad terraza fluvial. La recomendación de uso es: uso sin mayores restricciones, exceptuando los taludes que las limitan.

Figura $\mathrm{N}^{\circ} 6$

Zonificación de apoyo al ordenamiento territorial costero

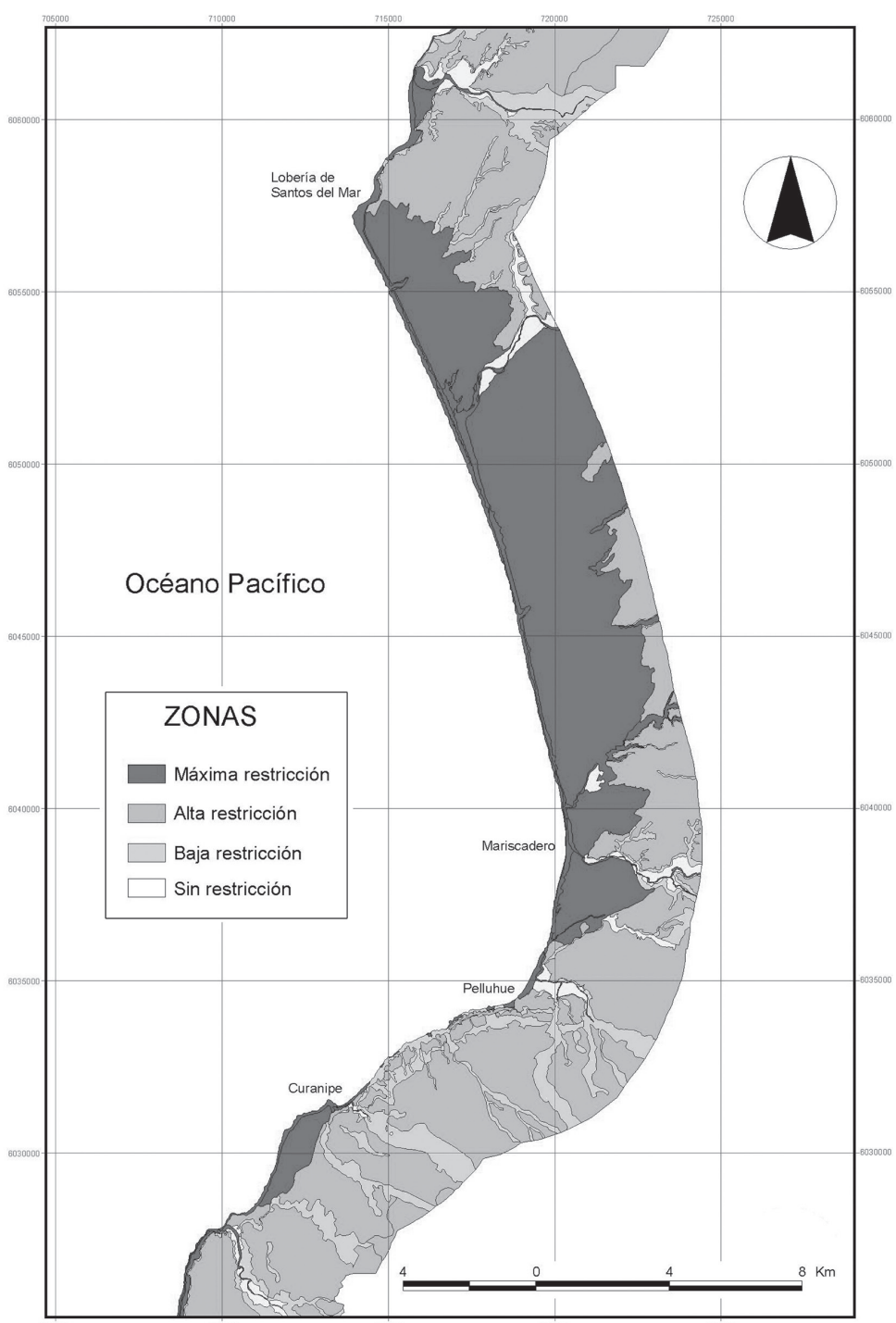

Fuente: Elaboración propia. 
Figura $\mathrm{N}^{\circ} 7$

Constitución: expansión urbana de vivienda social sobre unidad núcleo montañoso, con marcada modificación de las pendientes naturales

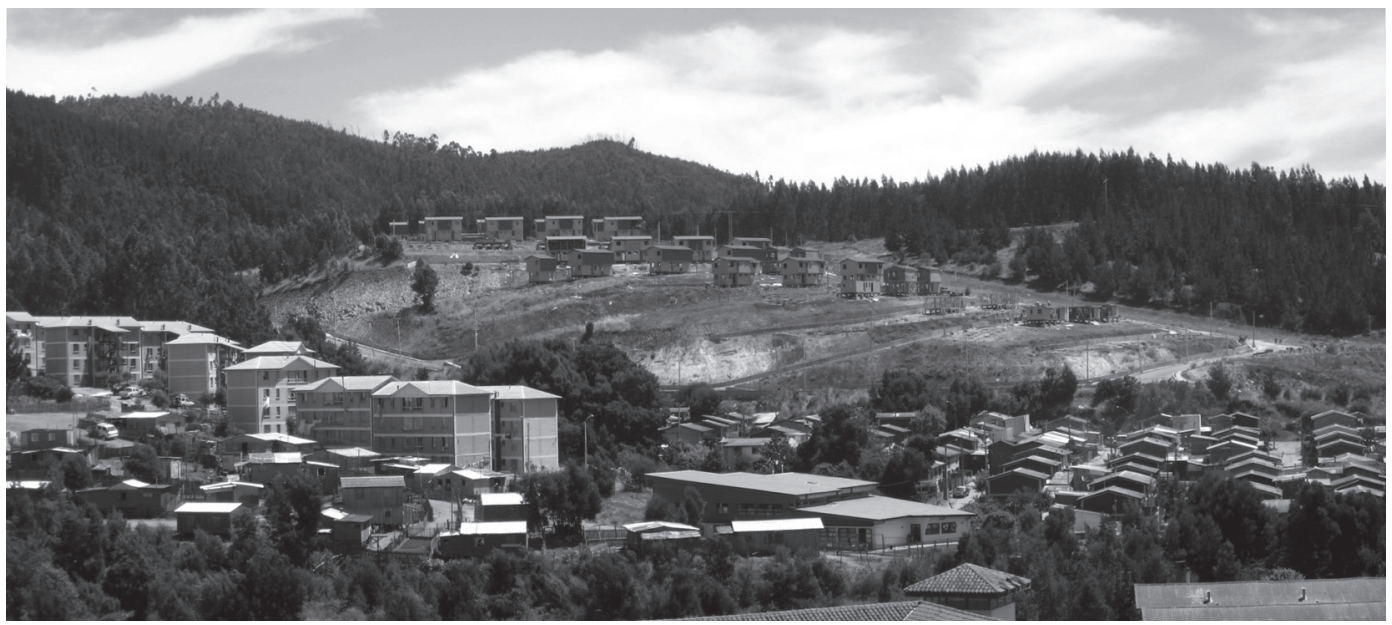

Fuente: Colección personal de los autores, 2008.

\section{Consideraciones finales}

El mejoramiento de la conectividad de la zona de estudio, en la medida que las condiciones de la economía chilena se recuperen, puede exponerla a crecientes presiones resultantes del aumento de la demanda por espacios de ocio y residencias secundarias. En este marco, el análisis de las condiciones de fragilidad que presenta la ZC de la Región del Maule adquiere especial relevancia pensando en que ciertos procesos de degradación pudieran acelerarse.

De acuerdo a los resultados de la investigación, más del $80 \%$ del área de estudio presenta grados de fragilidad alta y extrema, situación que impone considerar la condición natural del territorio frente a una eventual intensificación de la intervención antrópica sobre este medio costero sensible.

Con respecto a la situación de los IPT frente a las nuevas dinámicas de los procesos de cambio en el área de estudio, estos no están lo suficientemente adaptados a la naturaleza y velocidad de las transformaciones que ocurren, en particular las asociadas al desarrollo inmobiliario y a la ocupación industrial, como tampoco respecto de las ne- cesidades de protección de las zonas frágiles, a partir de criterios de mayor sensibilidad que los aplicados hasta ahora.

La propuesta de zonificación planteada en este trabajo busca sensibilizar y servir de apoyo a la elaboración de futuros instrumentos de planificación territorial o a nuevas actualizaciones, permitiendo desarrollos urbanos más armónicos con las condiciones naturales. Se requiere evitar situaciones de conflicto que surgen cuando el desarrollo urbano se realiza en unidades naturales sensibles al desencadenamiento de procesos morfogenéticos.

Probablemente este tipo de conflictos entre usos del suelo muy agresivos sobre unidades con niveles de fragilidad alta o extrema, requiera de medidas de mitigación que hasta el momento no se observan en la mayor parte de las zonas de extensión urbana en el área de estudio.

A partir de esta experiencia, queda planteado el desafío de proponer nuevas formas de utilización del espacio, más adecuadas a la diversidad de las condiciones de fragilidad del territorio. La geografía puede y debe contribuir a la incorporación de nuevos criterios que permitan mejorar las actuales herramientas de planificación territorial. 


\section{Referencias bibliográficas}

ANDRADE, B.; LAGOS, M. y ARENAS, F. Cuantificación de procesos morfogénicos actuales como indicador de la fragilidad de unidades fisiográficas en la costa de la Región de O'Higgins. Revista de Geografía Norte Grande, 2004, №31, p. 63-72.

ANDRADE, B.; ARENAS, F. y GUIJÓN, R. Revisión crítica del marco institucional y legal chileno de ordenamiento territorial: el caso de la zona costera. Revista de Geografía Norte Grande, 2008, N 41, p. 23-48.

BART, W. \& RUFF, L. Seismic coupling along the Chilean subduction zone. Journal of Geophysical Research, 1991, vol. 96, p. 11997-12022.

CENDRERO, A.; DÍAZ DE TERÁN, J. R. y FRANCÉS, E. Geología y ordenación de espacios litorales: análisis de casos. En: MARTÍNEZ, J. y CASAS, D. (eds.). Territorio litoral y su ordenación. Las Palmas de Gran Canaria: Serv. Publicaciones, Universidad de Las Palmas, 1989, p. 10-50.

CHARLIER, R. H. Costal zone: occupance, management and economic competitiviness. Ocean \& Shoreline Management, 1989, vol. 12, p. 383-402.

FARR, T. et al. The shuttle radar topography mission. Reviews of Geophysics., 2007, No 45, RG2004, doi: 10.1029/2005RG000183.

FITZ-ROY, R. Narrative of the surveying of H.M.S. "Adventure" and "Beagle" between the years 1826 and 1836 describing their examination of the southern shores of South America and the Beagle's circumnavigation of the globe. London: H. Coburn, 1839.

GOTO, C. \& OGAWA, Y. Numerical method of tsunami simulation with the leap-frog scheme. Tohoku: Dept. of Civil Engineering, Tohoku University. Translated for the TIME Project by N. Shuto, 1992.

GOTO, C.; OGAWA, Y.; SHUTO, N. \& IMAMURA, F. Numerical method of tsunami simulation with the leap-frog scheme. Paris: IUGG/IOC TIME Project Intergovernmental Oceanographic Commission of UNESCO, Manuals and Guides, №35, 1997.
GUIJÓN, R. Diagnóstico y aplicabilidad de las normas jurídicas de ordenamiento territorial en la zona costera de la Región del Maule. Seminario de grado licenciatura en Geografía. Santiago: Instituto de Geografía, Facultad de Historia, Geografía y Ciencia Política, Pontificia Universidad Católica de Chile, Chile, 2007.

IWASAKI, T. \& MANO, A. Two-dimensional numerical computation of tsunami run-ups in the Eulerian description. Tokyo: Twenty-sixth Conference on Coastal Engineering, 1979, p. 70-74.

JOLY, F. \& TRICART, J. Légénde de la carte géomorphologique de La France au 1:50.000. CNRS, RCP. 77. 1971, p. 78.

KAUSEL, E. y RAMÍREZ, D. Relaciones entre parámetros focales y macrosísmicos de grandes terremotos chilenos. Revista Geofísica, 1992, vol. 37, p. 36-194.

MANSHINHA, L. \& SMYLIE, D. The displacement field of inclined faults. Bulletin of the Seismological Society of America, 1971, vol. 61, No 5, p. 1433-1440.

SERVICIO HIDROGRÁFICO Y OCEANOGRÁFICO DE LA ARMADA DE CHILE (SHOA). Carta de inundación por tsunami Puerto Constitución, Chile. Valparaíso: SHOA, 2002.

SERVICIO HIDROGRÁFICO Y OCEANOGRÁFICO DE LA ARMADA DE CHILE (SHOA). Tablas de marea de la costa de Chile. Valparaíso: SHOA, 2008.

SMITH, W. \& SANDWELL, D. Seafloor topography from satellite altimetry and ship depth soundings. Science, 1997, vol. 277, p. 1957-1962.

VERSTAPPEN, H. TH. \& VAN ZUIDAM, R. A. Text of photo-interpretation. System of Geomorphological Survey. Delft: International Institute for Aerial Survey and Earth Sciences, vol. VII, 1968.

VIDAL DE LA BLACHE, P. Les genres de vie dans la géographie humaine. Annales de Géographie, 1911, p. 193-212 y 289-304. 